\title{
Planar liquid volume fraction and SMD distribution of Jatropha vegetable oil spray: effect of ethanol blending and GLR
}

\author{
ANIKET P KULKARNI and D DESHMUKH*(D) \\ Spray and Combustion Laboratory, Discipline of Mechanical Engineering, Indian Institute of Technology \\ Indore, Indore 453552, India \\ e-mail: dldeshmukh@iiti.ac.in
}

MS received 24 April 2018; revised 19 October 2018; accepted 22 October 2018; published online 1 February 2019

\begin{abstract}
This research paper presents the planar distribution of Sauter Mean Diameter (SMD) and liquid volume fraction in airblast sprays of Jatropha vegetable oil. The effect of ethanol blending at different gas to liquid mass ratios (GLRs) is presented. The planar SMD distributions are obtained using Structured Laser Illumination and Planar Imaging-Laser Sheet Drop sizing (SLIPI-LSD) and Particle/droplet Imaging Analysis (PDIA) techniques. The straight vegetable oil (SVO) spray showed poor atomization at GLR 1, which rose with increasing GLR. The blending of ethanol significantly improved the atomization of SVO even at GLR 1 condition. The liquid volume fraction distribution increased with higher GLR and with the increase in the percentage of ethanol in the blend. The impact of the increase in ethanol on drop size is small at GLR of 5. The E30 blend showed uniformly distributed liquid in the spray plane with uniform SMD distribution in the range of $50 \mu \mathrm{m}$ at GLR 5.
\end{abstract}

Keywords. SVO; ethanol blend; SLIPI-LSD; liquid volume fraction; SMD; GLR.

\section{Introduction}

The use of renewable fuels in combustion devices is ever increasing due to depletion of fossil fuels reserves, and stringent emission norms. The potential alternative biofuels for power generation include straight vegetable oils (SVOs), biodiesel, ethanol and methanol [1]. SVO is preferred in combustion devices due to lower cost compared with their biodiesel counterparts [2, 3]. Use of SVO avoids additional chemical processing and energy in the transesterification process. Hence use of SVO in oil-fired boilers, gas turbines and other stationary power generation devices is a preferable option [2,3]. However, the high viscosity of SVO is a hurdle in practical implementation [4, 5]. The high viscosity of oils results in poor atomization and hence poor combustion performance [6]. Atomization plays an essential role in determining the combustion efficiency and emissions of liquid-fuelled combustion chambers [7]. Hence, it is vital to study atomization of SVOs in stationary combustion devices. Fan et al [8] studied spray characteristics of Jatropha SVO with a pressure swirl atomizer using a diffraction-based method. They observed improved atomization of SVO using assist-air. Sivakumar et al [9] studied spray characteristics of Jatropha biofuels and its blends with Jet A fuel in a simplex swirl atomizer. They observed that the spray characteristics of Jatropha biofuel

*For correspondence were comparable to those of Jet A-1 fuel. Stationary power generation devices prefer twin-fluid atomization strategy over pressure swirl atomization due to the lower injection pressure of the liquid in the twin-fluid atomization [10]. Basak et al [11] reported that air-assist atomization required lower pumping power compared with that of the pressure-swirl atomization. Air-assist atomizers use a small amount air at high velocity for atomization of a liquid. Avulapati and Ravikrishna [12] used the air-assisted impinging jet for atomization of Jatropha oil to achieve Sauter Mean Diameter (SMD) of $50 \mu \mathrm{m}$ at gas to liquid mass ratio (GLR) below 0.1 . Lujaji et al [13] studied spray characteristics of bio-oil with ethanol blends using externally mixed air-assist nozzles. It was shown that SMD less than $100 \mu \mathrm{m}$ can be achieved with $40 \%$ addition of ethanol to bio-oil at 30 SLPM atomization air flow rate. Airblast atomizers use a significant amount of air at low velocity. The presence of the higher mass of air in the airblast atomization can help improve combustion and reduce $\mathrm{CO}$ emissions from a stationary combustion device. Simmons and Agrawal [14] performed drop size and velocity measurements using Phase Doppler Anemometry (PDA) in soybean oil sprays with flow-burring airblast atomization. They observed a peak SMD of $54 \mu \mathrm{m}$ at $20 \mathrm{~mm}$ below the nozzle tip along the spray axis for GLR 2. Overall, various atomization strategies have been implemented to study spray characteristics of different SVO feed-stocks for combustion applications. Jatropha, which is a non-edible 
feed-stock, has no impact on food resources unlike many other edible biofuels and is preferred as renewable fuel at many parts of the world. Hence, it is important to study spray characteristics of Jatropha SVO in airblast atomization, which is a widely used atomization strategy in stationary combustion devices.

The non-uniformity in the spatial distribution of SMD has a direct impact on ignition delay and combustion efficiency $[15,16]$. Further, liquid volume fraction distribution can be considered as an indication of temperature distribution [10]. There are no data available in the literature on the planar distribution of SMD and liquid volume fraction for vegetable oil sprays. The planar SMD distribution and liquid volume fraction distribution together provide essential inputs for design and retrofitting of combustors of stationary combustion devices.

The viscosity of the SVO can be reduced by preheating the oil or blending it with a liquid of lower viscosity such as ethanol, methanol or diesel. Panchasara and Agrawal [17] studied airblast atomization of preheated Soybean SVO. They reported SMD of $30 \mu \mathrm{m}$ at GLR 4 with SVO preheated to $80{ }^{\circ} \mathrm{C}$. However, preheating of SVO needs extra energy input, which may reduce the net power output of the combustion device. To mitigate this issue, blending of ethanol or diesel with SVO can be a probable solution. Ethanol, which is a renewable fuel, is preferred over diesel.

This study focuses on the effect of ethanol blending with Jatropha SVO on planar drop size and liquid volume fraction distributions in an airblast spray. The planar SMD distribution is obtained using Structured Laser Illumination and Planar Imaging-Laser Sheet Drop sizing (SLIPI-LSD) technique [18]. The liquid volume fraction in the spray plane is obtained using SLIPI-PLIF (Planar laser Induced Fluorescence) and Particle/droplet Imaging Analysis (PDIA) techniques. The drop size distributions are obtained using the PDIA technique at the edge of the spray for various GLR conditions.

\section{Experimental set-up}

The experimental set-up for PDIA and SLIPI-LSD measurements is shown in figure 1. An Nd:YAG pulsed laser ( $\lambda=532 \mathrm{~nm}, 200 \mathrm{~mJ}$ maximum energy, $10 \mathrm{~ns}$ pulse-width) is used as an illumination source in both measurements. The PDIA set-up consists of the pulsed laser and a longdistance microscope (Questar, QM-1) with a CCD camera (PCO Sensicam). A bright background required for microscopic shadowgraphy is obtained using an in-housedeveloped optical diffuser [19]. The optical diffuser, based on the design proposed by Jain et al [19], consists of a spherical concave lens (Holmarc, $80 \mathrm{~mm}$ focal length), a diffuser (Holmarc, HO-DF-100S-15) and a cuvette filled with rhodamine $6 \mathrm{G}$ solution (concentration of $10 \mathrm{mg}$ per litre in distilled water). The laser beam is expanded using the spherical concave lens and incident on the diffuser attached to a wall of the cuvette. The expanded and diffused laser beam then illuminates the rhodamine solution. Fluorescence is generated in the cuvette due to excitation of the rhodamine dye with the laser. Thus, a speckle-free and uniform background is obtained. A field of view for the droplet size measurement is $1.92 \mathrm{~mm} \times 1.44 \mathrm{~mm}$ with a resolution of $1.2 \mu \mathrm{m} /$ pixel [20]. A large number of images (more than 330) are captured in the PDIA measurements to ensure a statistically large number of droplets in the measurement location. These microscopic shadowgraphic images are further analysed using an image processing algorithm to get SMD, drop-size distribution and liquid volume fraction at a point [21]. The depth of field (DOF) of the optical system is measured using a Patterson Globe. The Patterson Globe is moved in steps of $50 \mu \mathrm{m}$ from either side of the focal plane to determine DOF correction factor for various diameters [22]. The resolution of the PDIA technique is limited by the diffraction limit of the optical system and a minimum number of pixels per droplet [23, 24]. The minimum droplet diameter detectable with the PDIA system is kept at $7 \mu \mathrm{m}$ in the present work. This avoids diffraction limit and also measures droplets with acceptable accuracy. Droplets with a diameter less than $7 \mu \mathrm{m}$ are neglected in the SMD calculations.

Mie and PLIF signals are acquired using the SLIPI technique to reduce the contribution of multiple scattering. The experimental set-up for SLIPI measurements consists of a pulsed laser, sheet making optics, a Ronchi grating, a bandpass filter (Edmund Optics, OD 6, centred at $560 \mathrm{~nm}$ with $32 \mathrm{~nm}$ full-width at half-maximum (FWHM) and the CCD camera. The field of view of the camera with a macrolens in planar imaging is $88 \mathrm{~mm} \times 55 \mathrm{~mm}$ with a resolution of $88.5 \mu \mathrm{m} /$ pixel. A structured laser sheet is generated using an in-house-developed Ronchi grating of $5 \mathrm{lp} / \mathrm{mm}$. The Ronchi grating is developed from a $500 \mathrm{~nm}$ thick aluminium coating on a glass slide. A photo-resist mask is prepared using UV lithography. A wet etchant (acetic acid $(10 \mathrm{ml})$, phosphoric acid $(75 \mathrm{ml})$, water $(10 \mathrm{ml})$ and nitric acid $(1-2 \mathrm{ml})$ ) is used to develop the grating of $5 \mathrm{lp} /$ $\mathrm{mm}[25,26]$. The spatial modulation of the structured laser sheet is obtained by moving the Ronchi grating in a vertical direction using a translation stage. The harmonics, generated due to diffraction of laser light in the grating, are removed using a frequency cutter $[27,28]$. Rhodamine $6 \mathrm{G}$ is used as a fluorescence dye with the concentration of 30 mg per litre of SVO. A commercially available airblast atomizer from Delavan (30609-2) is used in the present work. Stationary power generation devices such as furnaces, boilers and room heating units extensively use these atomizers. The atomizer is externally mixed with the liquid jet diameter of $0.3 \mathrm{~mm}$ and uses swirling atomizing air for atomization of the liquid. The experiments are performed under non-evaporative ambient conditions. The liquid flow rate is kept constant at $12 \mathrm{ml}$ per minute. The air flow rate is 

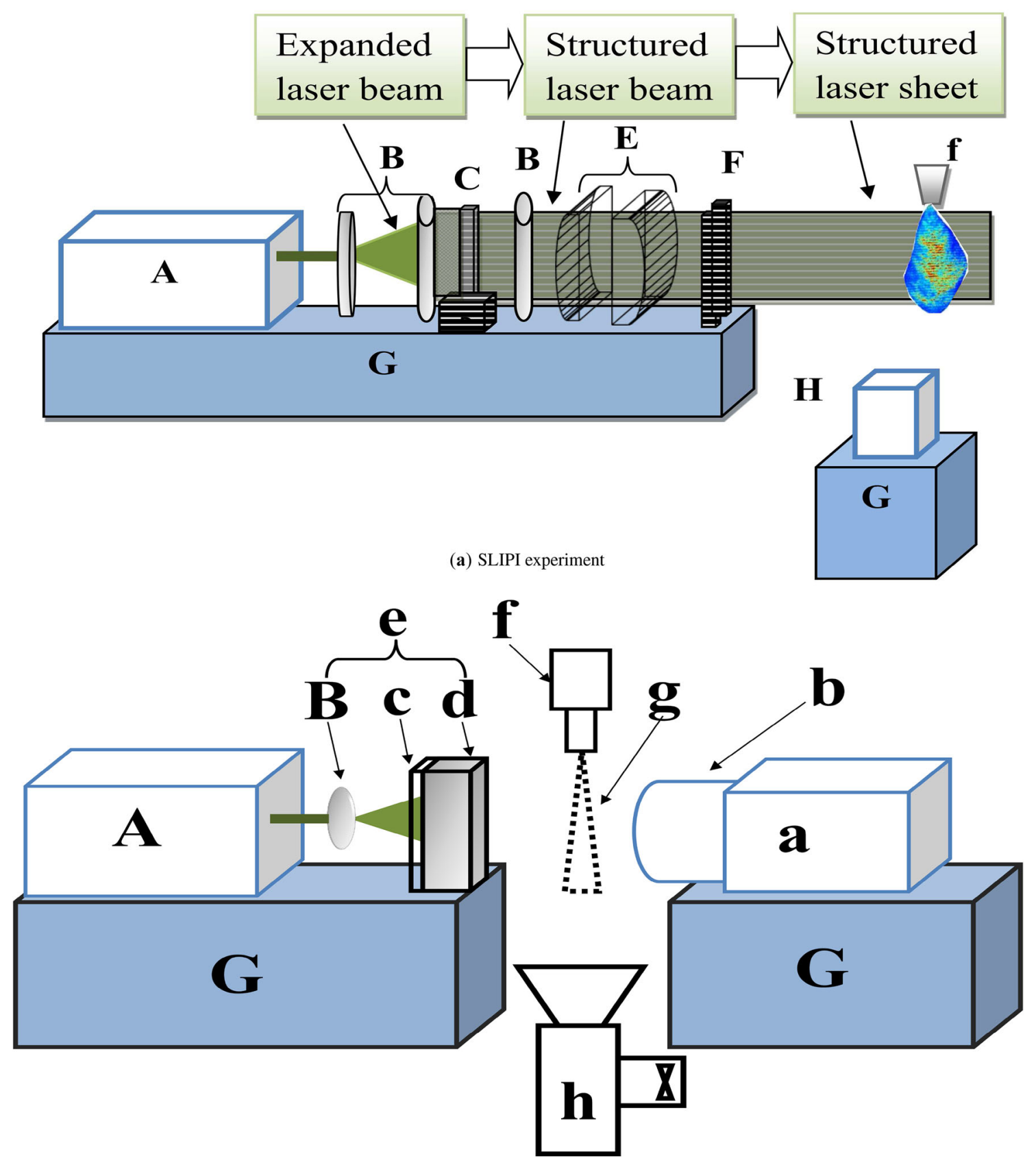

(b) PDIA experiment

Figure 1. An optical arrangement for SLIPI-LSD measurements. (A) Nd:YAG laser. (B) Spherical convex lens. (C) Ronchi grating. (D) $X-Y-Z$ stage. (E) Pair of cylindrical lenses. (F) Frequency cutter. (H) CCD camera with macro-lens. (G) Optical table. (a) CCD camera. (b) Long-distance microscope. (c) Diffuser glass. (d) Cuvette with Rhodamine 6G solution. (e) Optical diffuser arrangement. (f) Airblast atomizer. (g) Airblast spray. (h) Spray collection device.

varied to achieve GLR of 1, 3 and 5. The liquid flow and air flow rates are controlled using a liquid mass flow controller (Bronkhorst M14, the accuracy of $\pm 0.2 \%$ ) and an air flow controller (Aalborg GFC 57C, the accuracy of $\pm 3 \%$ ), respectively.

The planar SMD distribution in the spray is obtained using a ratio of PLIF and Mie signals. The calibration constant in the LSD technique is obtained using SMD from the PDIA technique along the edge of the spray as shown in figure 2. The Mie and PLIF imaging suffers from error due to multiple scattering of the laser sheet and the signals. This error in the Mie and PLIF imaging is corrected using the SLIPI technique and numerical methods [27-30]. Three spatially modulated images $\left(\mathrm{I}_{1}, \mathrm{I}_{2}\right.$ and $\left.\mathrm{I}_{3}\right)$ are obtained by 


\section{A P Kulkarni and D Deshmukh}

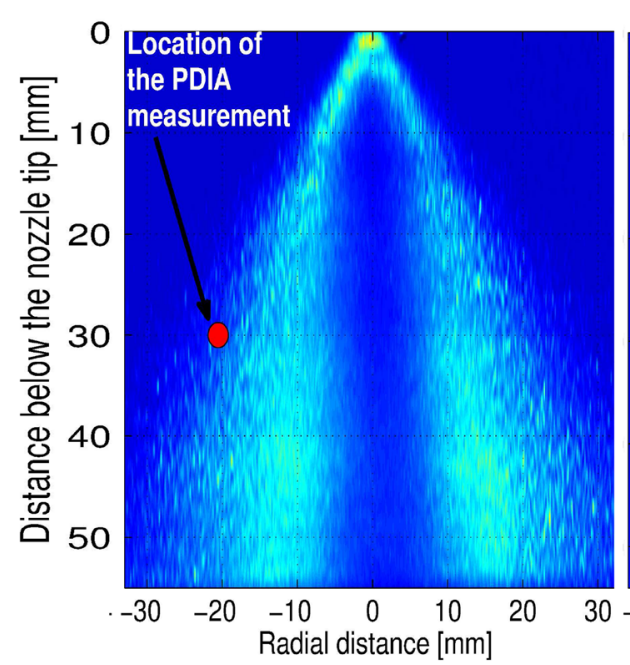

(a) GLR 1

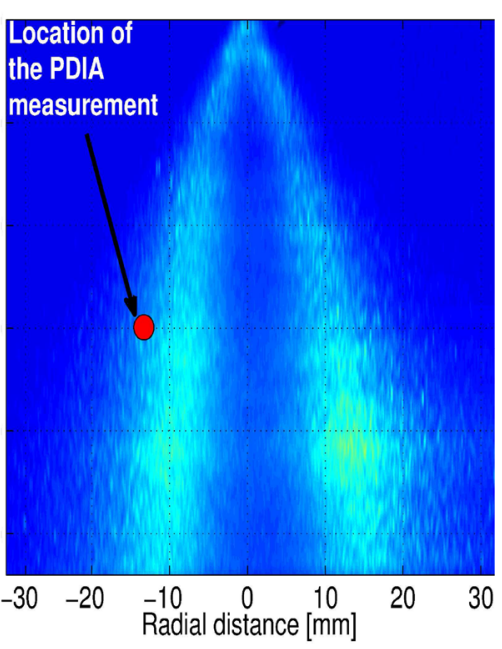

(b) GLR 3

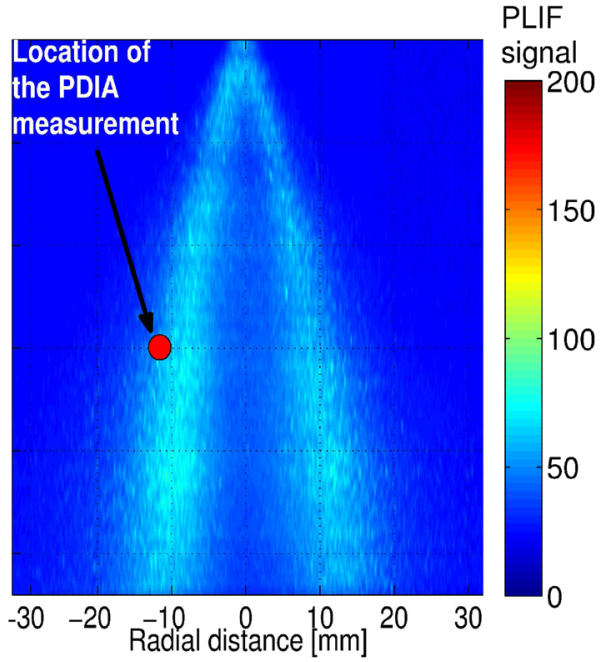

(c) GLR 5

Figure 2. PLIF signals of SVO at GLR 1, 3 and 5.

moving the Ronchi grating in a vertical direction with a period of one-third of the modulation.

A SLIPI image is obtained using Eq. (1):

$$
I_{S L I P I}=\frac{\sqrt{2}}{3}\left[\left(I_{1}-I_{2}\right)^{2}+\left(I_{2}-I_{3}\right)^{2}+\left(I_{3}-I_{1}\right)^{2}\right]^{1 / 2} .
$$

Final Mie and PLIF images are obtained by averaging 150 SLIPI images of the respective signals. Numerical corrections are applied to overcome losses due to scattering and absorption of the laser sheet [25, 31-33]. Liquid volume fraction measurements are obtained using the corrected PLIF images along with the PDIA technique [25, 33]. The uncertainty in SMD measurement and liquid volume fraction measurements is estimated as $9 \%$ and $21 \%$, respectively [20, 34].

Ethanol blends of Jatropha SVO are prepared by adding ethanol in Jatropha oil on a volumetric basis. Three ethanol blends, E10 (90\% SVO and 10\% ethanol), E20 (80\% SVO and $20 \%$ ethanol) and E30 (70\% SVO and 30\% ethanol), are used. The properties of the test fuels, measured at room temperature of $25{ }^{\circ} \mathrm{C}$, are compared in table 1 . Kinematic viscosity is measured using a Redwood viscometer (type 1),

Table 1. Properties of the test fuels.

\begin{tabular}{lccc}
\hline Fuel & $\begin{array}{c}\text { Density } \\
\left(\mathrm{kg} / \mathrm{m}^{3}\right)\end{array}$ & $\begin{array}{c}\text { Kinematic viscosity } \\
(\mathrm{cSt})\end{array}$ & $\begin{array}{c}\text { Surface tension } \\
(\mathrm{mN} / \mathrm{m})\end{array}$ \\
\hline SVO & 915 & 54 & 32 \\
E10 & 904 & 48 & 31 \\
E20 & 892 & 42 & 30 \\
E30 & 880 & 37 & 29 \\
\hline
\end{tabular}

and density is measured using a digital mass balance (CITIZEN Semi-Micro-Balance CX Series, accuracy of $0.01 \mathrm{mg}$ ). Surface tension is calculated using the mixture rule and surface tension of ethanol [35] and Jatropha SVO [36]. The accuracy of the measurements is confirmed by performing measurements of water and ethanol as standard liquids. The blending of Jatropha SVO with ethanol reduces the viscosity of the blend up to $35 \%$. The density of the blend decreased marginally up to $3 \%$ for E30 blend. The viscosity plays an important role in atomization of a liquid. Hence, the atomization of the SVO is expected to improve with the blending of ethanol.

\section{Results and discussion}

The Mie and PLIF images are used to obtain planar SMD, and planar liquid volume fraction distribution in the spray plane using the PDIA technique. The effect of ethanol blending in Jatropha SVO on drop size distribution, planar SMD and liquid volume fraction distributions is studied at various GLR conditions.

\subsection{Spray structure}

The averaged PLIF images of SVO are compared in figure 2 to study the spray structure of SVO spray at GLR 1, 3 and 5. All the GLR conditions show a hollow cone spray structure. The spray cone angle is measured as an angle between tangents to the spray edges near the exit orifice $[7,20]$. The spray cone angle is determined at $15 \mathrm{~mm}$ 
below the nozzle tip using an averaged PLIF image. The spray is wide at GLR 1 with spray cone angle of $82^{\circ}$. The spray cone angle reduced from $63^{\circ}$ at GLR 3 to $56^{\circ}$ at GLR 5. This may be due to the increase in the axial velocity of the atomizing air with the increase in GLR [37]. The spray cone angles of the blended fuels are similar to those of SVO, showing a negligible effect of ethanol addition. GLR has a more significant impact on the spray cone angle than the ethanol addition.

\subsection{Drop size distribution}

The droplet size distributions at the edge of the spray are obtained using the PDIA technique. The drop size distributions for the test fuels at GLR 1, 3 and 5 are compared in figure 3. The SVO spray shows a large number of big droplets with diameter greater than $80 \mu \mathrm{m}$ at GLR 1 . These big droplets are undesirable in a combustion chamber, as they may lead to incomplete combustion and higher emissions. The percentage of the number of droplets with the diameter less than $20 \mu \mathrm{m}$ is lower for GLR 1, which shows
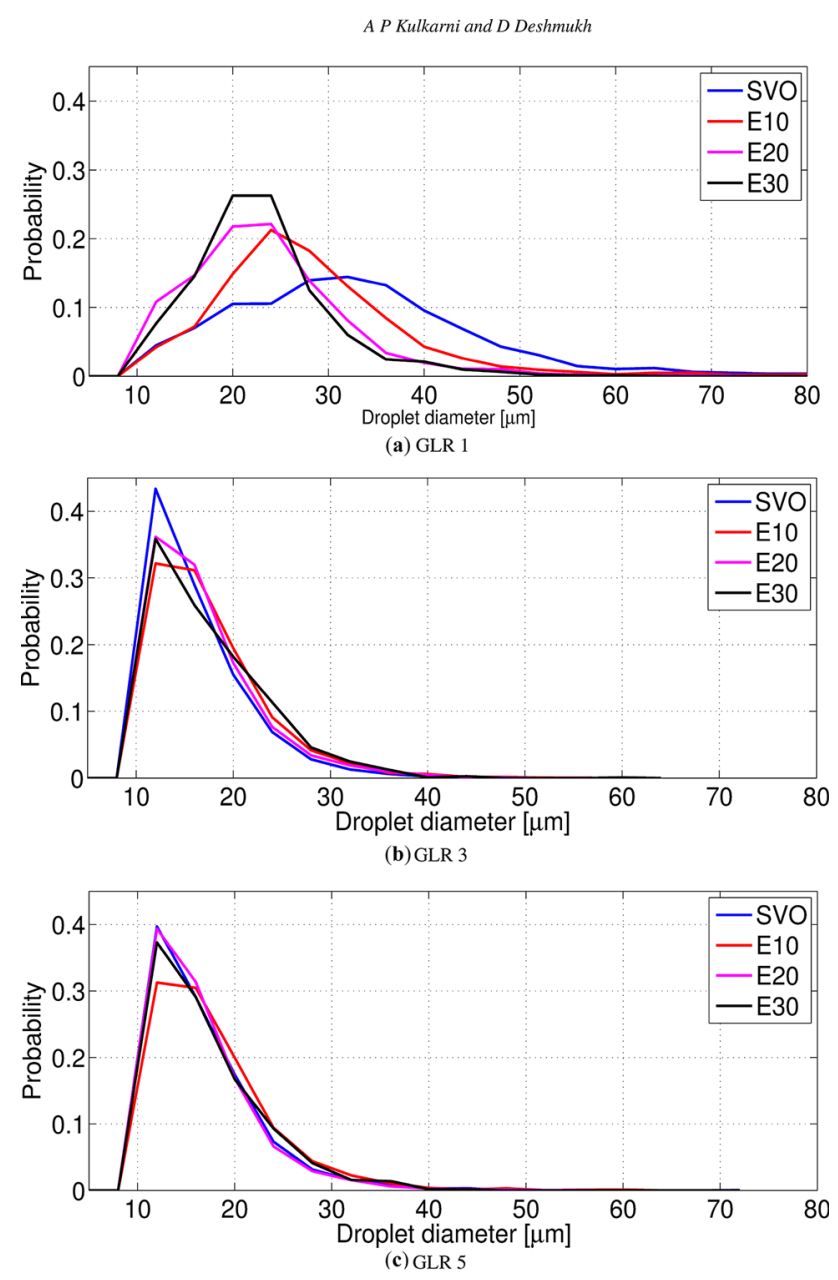

Figure 3. Drop size distributions at $30 \mathrm{~mm}$ below the nozzle tip. poor atomization of SVO. The high viscosity of the SVO may be responsible for the poor atomization. The fuels blended with ethanol show a large number of small droplets compared with SVO. The E10 blend gives peak droplet diameter at $25 \mu \mathrm{m}$ compared with $32 \mu \mathrm{m}$ for SVO. The number of droplets with the diameter greater than $80 \mu \mathrm{m}$ has decreased significantly. It is interesting to note that $10 \%$ addition of ethanol in SVO improves the atomization considerably while viscosity decreases by $11 \%$. The drop size distribution shifted towards smaller drop size with the increase in the percentage of ethanol in SVO blend. This shows improved atomization of ethanol blended fuels at GLR 1 due to the decrease in viscosity of blends. It is observed that the effect of ethanol blending is diminishing at high GLR conditions. The droplet size distribution at GLR of 5 is similar for SVO and all blends.

The droplet size distribution becomes narrower with the increase in GLR from 1 to 3 and 5. When GLR is increased to 3 , a large number of small droplets are observed. The largest droplet diameter reduced to $75 \mu \mathrm{m}$ from $150 \mu \mathrm{m}$. This suggests improved atomization at GLR 3 and 5, which is due to increase in kinetic energy of the atomizing air. The peak droplet diameter is around $15 \mu \mathrm{m}$ for both GLR 3 and 5. This indicates that increasing GLR beyond 3 may not help in droplet break-up.

\subsection{Radial SMD profiles}

Radial SMD profiles of SVO and its ethanol blends are compared in figure 4 for GLR 1, 3 and 5. The radial SMD profiles are obtained using the SLIPI-LSD technique at 30 $\mathrm{mm}$ below the nozzle tip. Higher SMD values are observed along the spray periphery for all measurement conditions. This is because of swirling atomizing air, which carries bigger droplets to the spray periphery due to centrifugal force. The highest SMD value $(\sim 150 \mu \mathrm{m})$ is observed for GLR 1 at a spray periphery for SVO fuel. The addition of ethanol reduced SMD values considerably, and the maximum SMD value is $\sim 60 \mu \mathrm{m}$ for E30 at GLR 1. The atomizer might work in the classical mode of atomization at low GLR conditions, where the viscosity of the liquid has a strong control over the atomization process [38]. The kinematic viscosity of the SVO is reduced with the addition of ethanol, which might have improved atomization of E30 at GLR 1.

An increase in GLR led to the reduction in SMD values for all test fuels. At GLR 3, the highest SMD value is observed to be $75 \mu \mathrm{m}$ for SVO. Ethanol-blended fuels showed further reduction with maximum SMD of $\sim 50 \mu \mathrm{m}$ for E30 at GLR 3. The difference in SMD of SVO and E30 is comparatively lower $(\sim 25 \mu \mathrm{m})$ at the spray periphery. When GLR is increased further (GLR 5), the highest SMD value $(\sim 55 \mu \mathrm{m})$ is considerably lower. Addition of ethanol leads to further reduction in SMD, and maximum SMD of $\sim 40 \mu \mathrm{m}$ is observed along the spray periphery for E30 


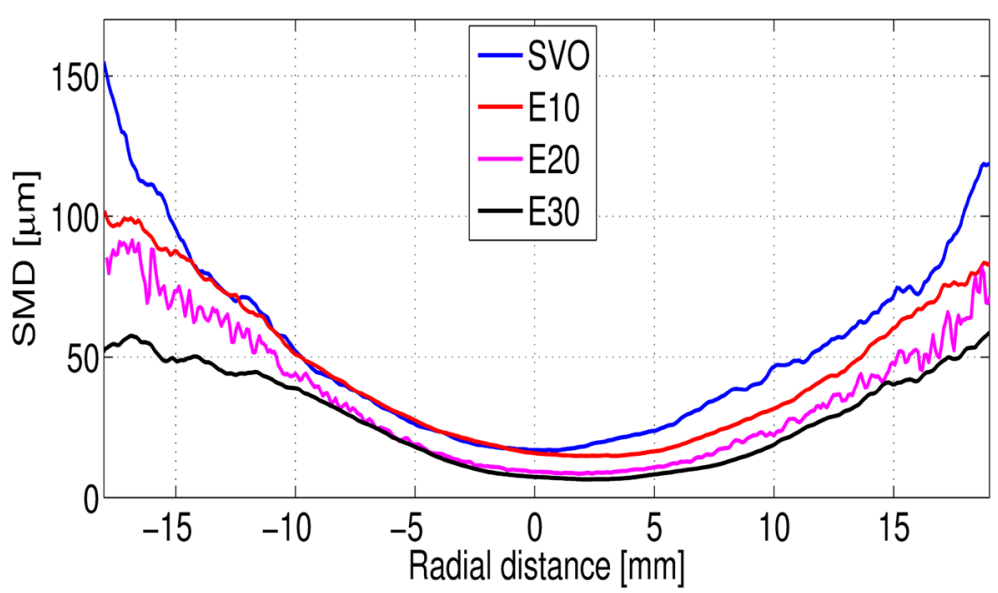

(a) GLR 1

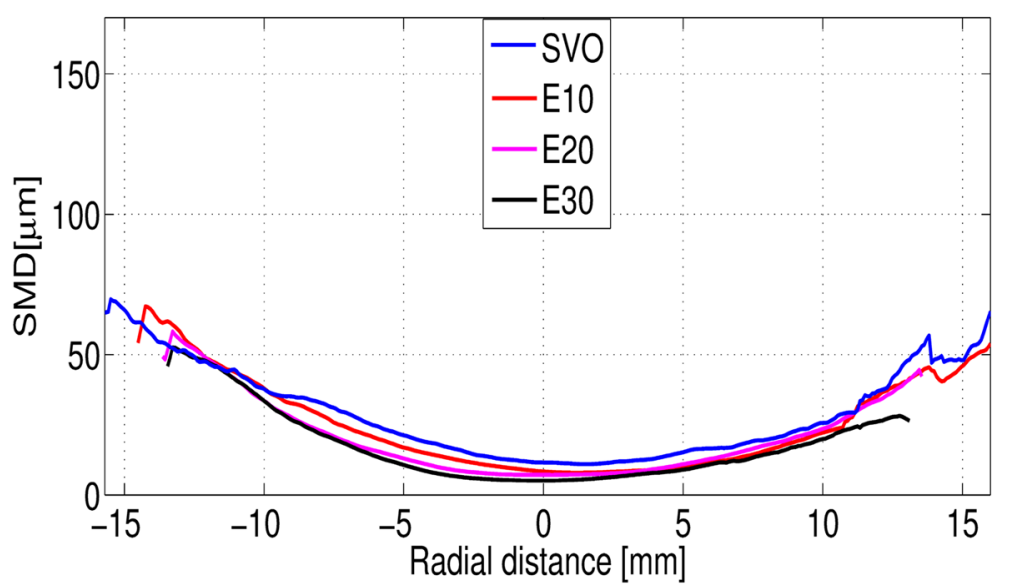

(b) GLR 3

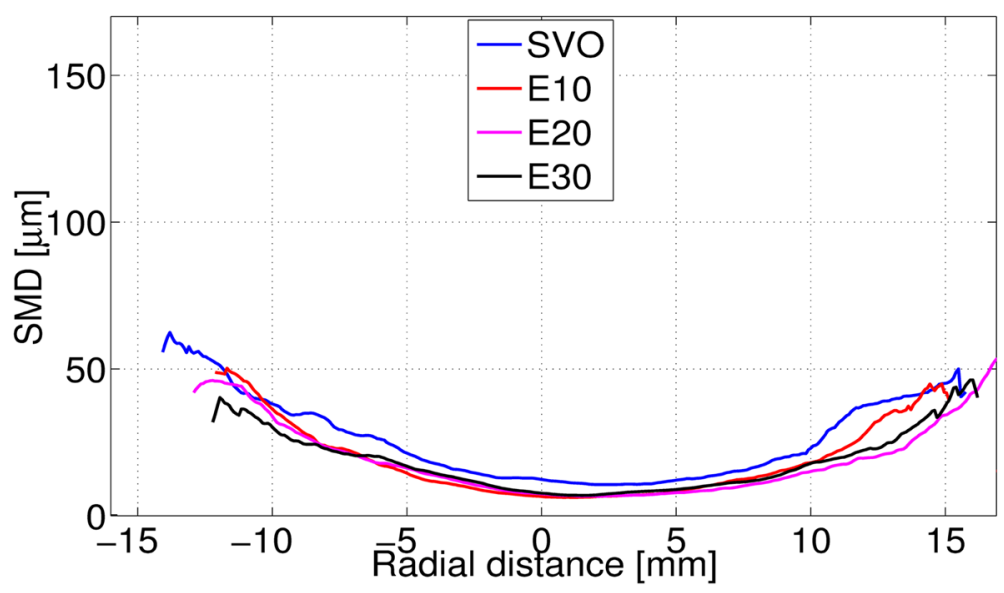

(c) GLR 5

Figure 4. Radial SMD profiles at $30 \mathrm{~mm}$ below the nozzle tip.

at GLR 5. The effect of the addition of ethanol to the SVO is marginal on the maximum SMD at high GLR conditions. The difference in the maximum SMD values of SVO and E30 is $\sim 15 \mu \mathrm{m}$. This might be attributed to prompt atomization at high GLR conditions [38]. The SMD in the range of 40-55 $\mu \mathrm{m}$ can be achieved for SVO and its ethanol blends by operating this atomizer at GLR 5. The increase in GLR and the addition of ethanol led to the reduction in 
SMD values due to improved atomization. The atomization of viscous SVO at low GLR conditions can be improved with $30 \%$ ethanol blending.

\subsection{Planar SMD distributions}

Planar SMD distributions of SVO and ethanol blends are compared in figure 5. The planar SMD distributions are obtained using SLIPI-LSD and PDIA techniques. SVO fuel showed very high SMD values along the spray periphery at GLR 1 due to its poor atomization. This suggests uneven distribution of the SVO fuel at GLR 1, which may lead to rich and lean pockets of the air-fuel mixture in a combustor.

Addition of ethanol to the SVO improved the SMD distribution at all GLR conditions. E30 fuel showed more uniform SMD distribution than that of SVO. When GLR is increased, SMD distribution is improved for all fuels, showing the reduction in SMD values. SVO showed improved SMD distribution at GLR 5 compared with GLR 1. E30 fuel showed the lowest SMD along the spray periphery $(\sim 40 \mu \mathrm{m})$ with uniform SMD distribution. A uniform SMD distribution is obtained for E30 fuel at GLR 5 , which may lead to uniform air-fuel mixture formation.

\subsection{Radial liquid volume fraction profiles}

Planar liquid volume fraction distribution is obtained using SLIPI-PLIF and liquid volume fraction at a point from the PDIA technique [22, 25, 33]. The fluorescence signal is proportional to the volume of the liquid $\left(V_{F}\right)$ present in the total pixel volume $\left(V_{T}\right)[31,33]$. The equivalent density $\rho_{e}$ is defined to consider two-phase nature of a spray as given in the following equation:

\section{A P Kulkarni and D Deshmukh}

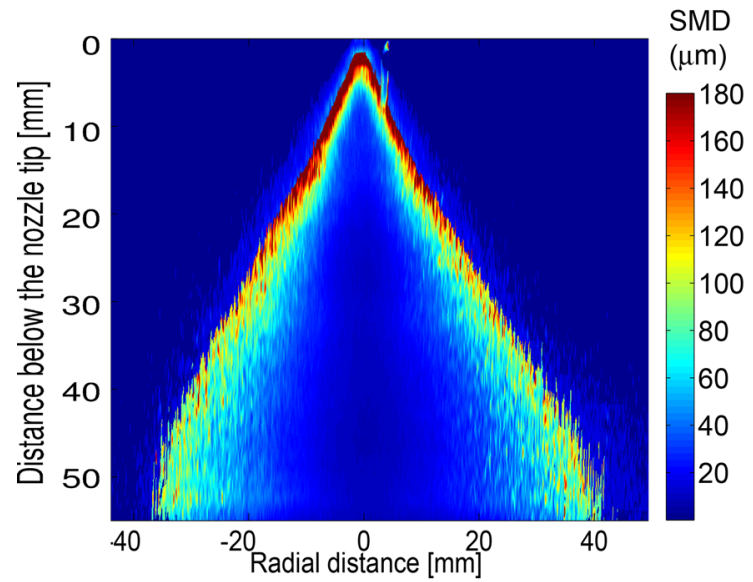

(a) SVO at GLR1

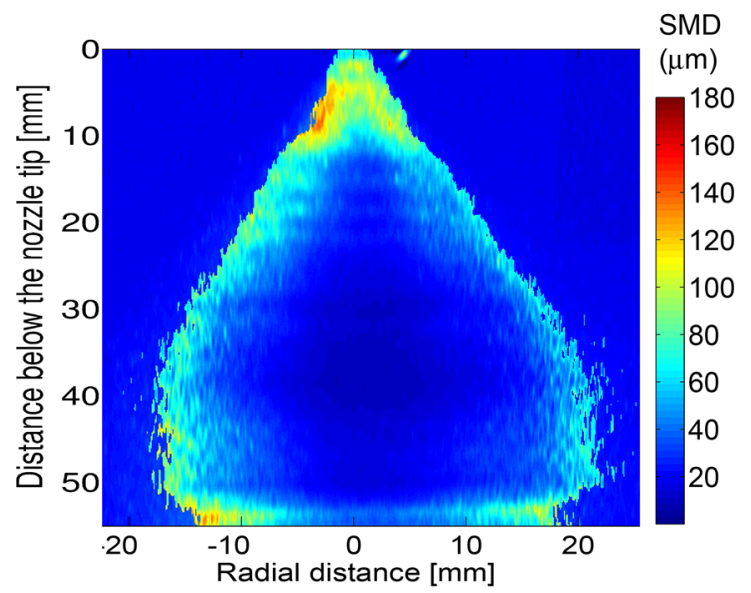

(c) SVO at GLR 5

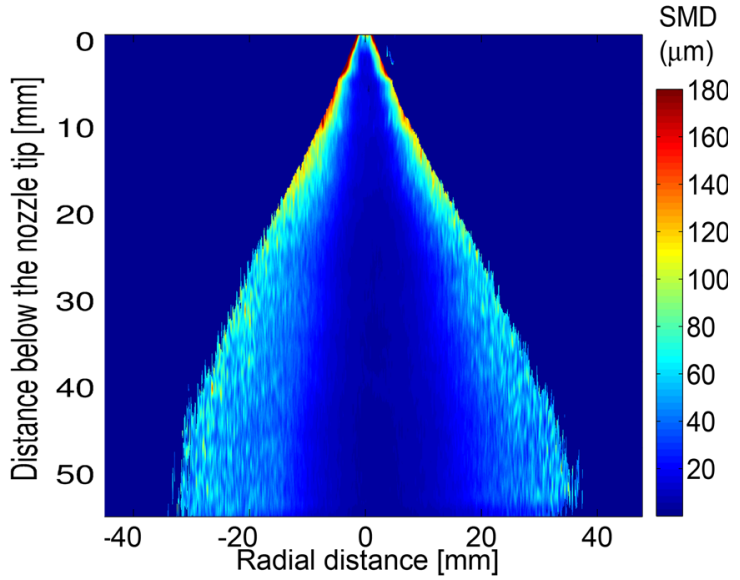

(b) E30 at GLR 1

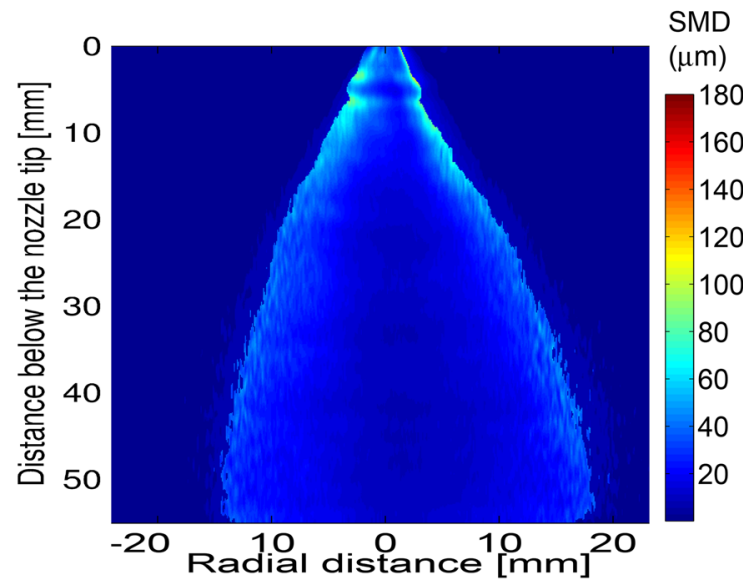

(d) E30 at GLR 5

Figure 5. Planar SMD distributions of SVO and E30 fuels at GLR 1 and 5. 


\section{A P Kulkarni and D Deshmukh}

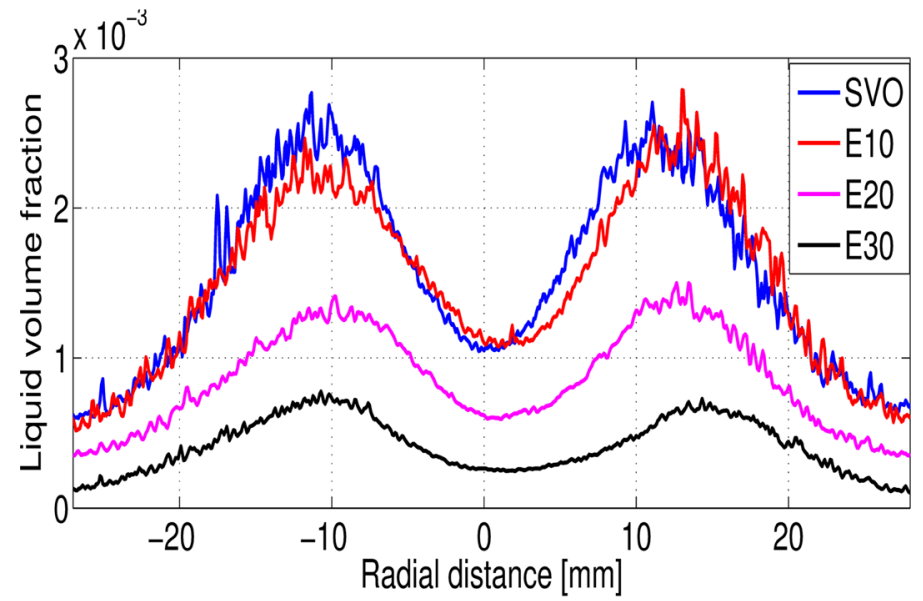

(a) GLR 1

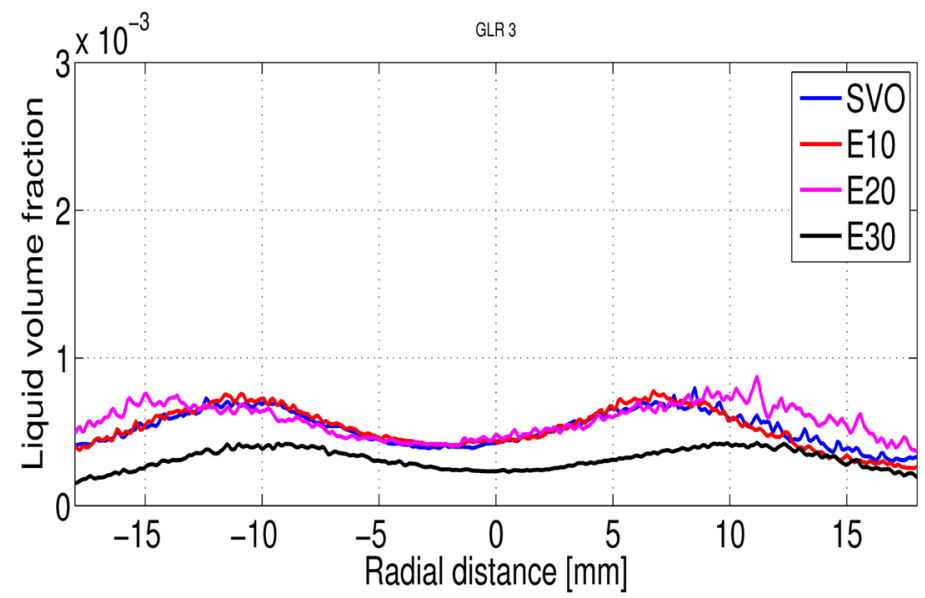

(b) GLR 3

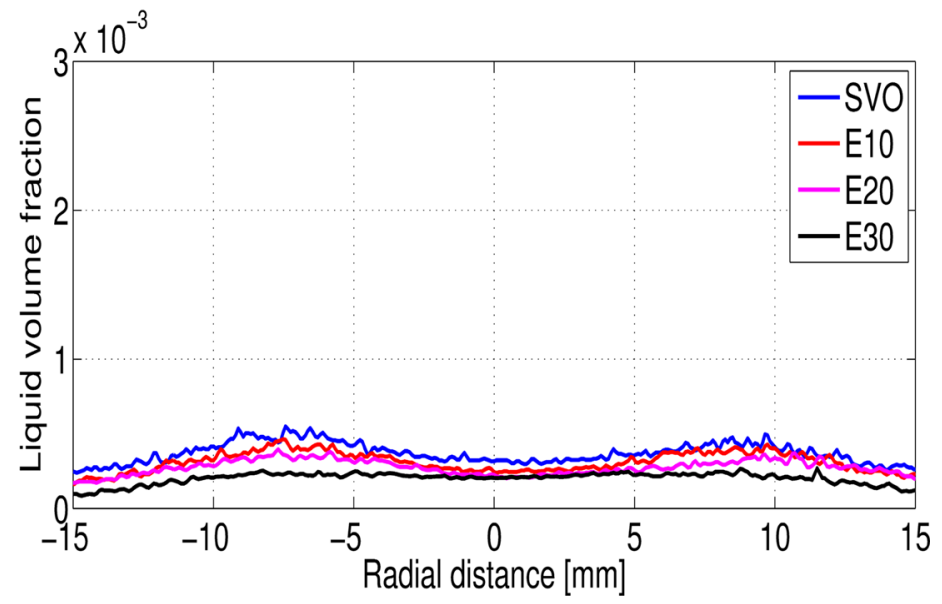

(c) GLR 5

Figure 6. Liquid volume fraction profiles at $30 \mathrm{~mm}$ below the nozzle tip.

$$
\rho_{e}=\rho \frac{V_{F}}{V_{T}}
$$

where $\rho$ is the density of a liquid.

Thus, the fluorescence signal in terms of $\rho_{e}$ is given as

$$
S_{F}(x, y)=K_{1} \rho_{e}(x, y)
$$

where $S_{F}(\mathrm{x}, \mathrm{y})$ is SLIPI-PLIF signal at a pixel $(x, y)$ and $\rho_{e}$ is equivalent density. The constant $\left(K_{1}\right)$ is determined using the PDIA technique by calculating the total volume of 


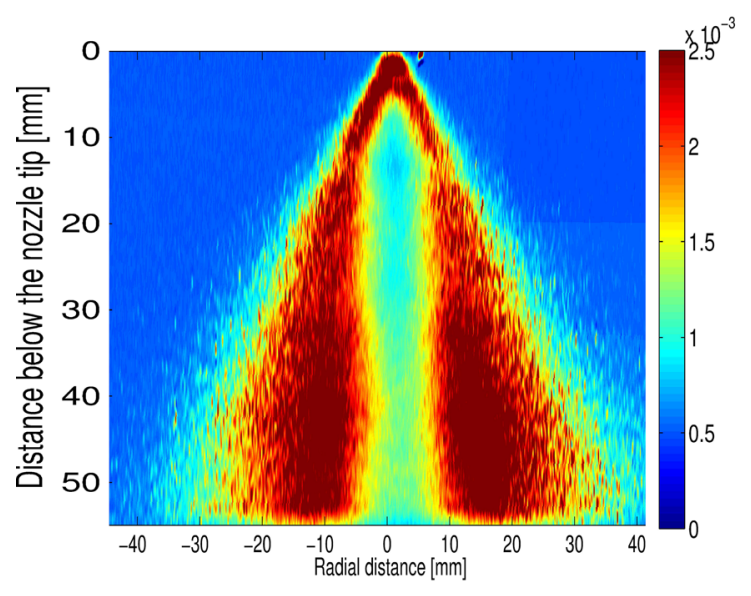

(a) SVO GLR1

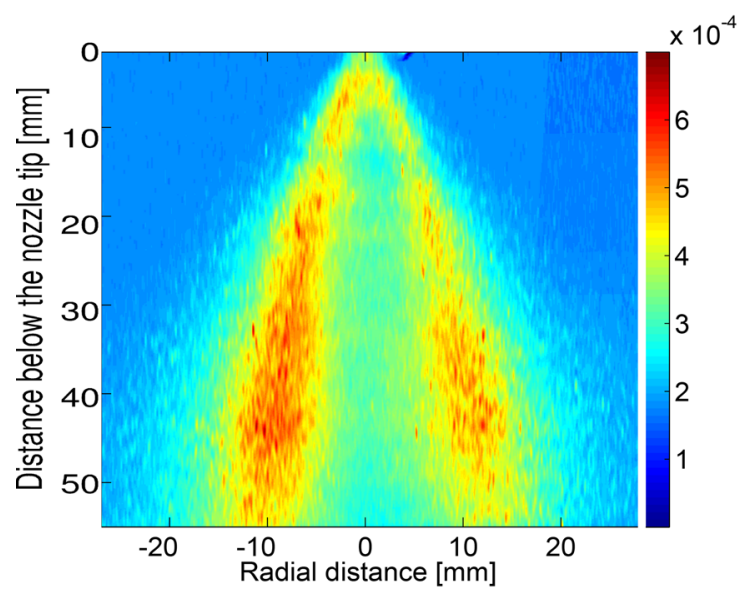

(c) SVO GLR5

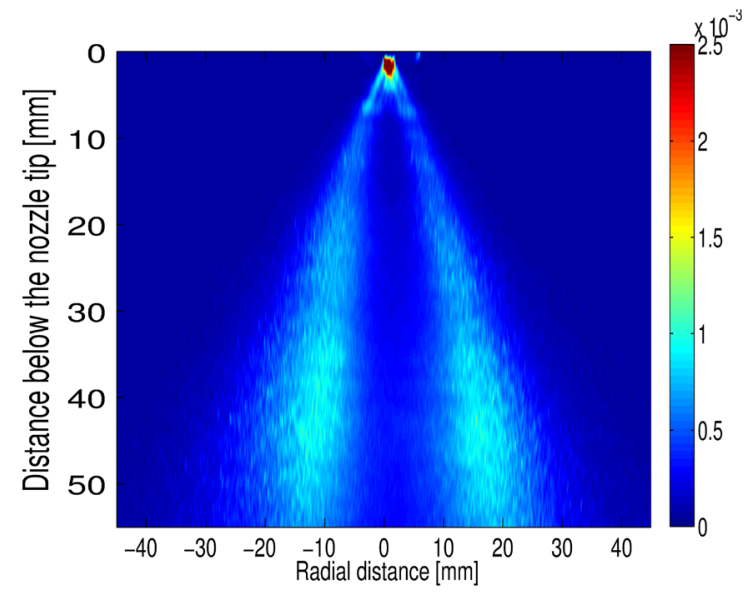

(b) E30 GLR 1

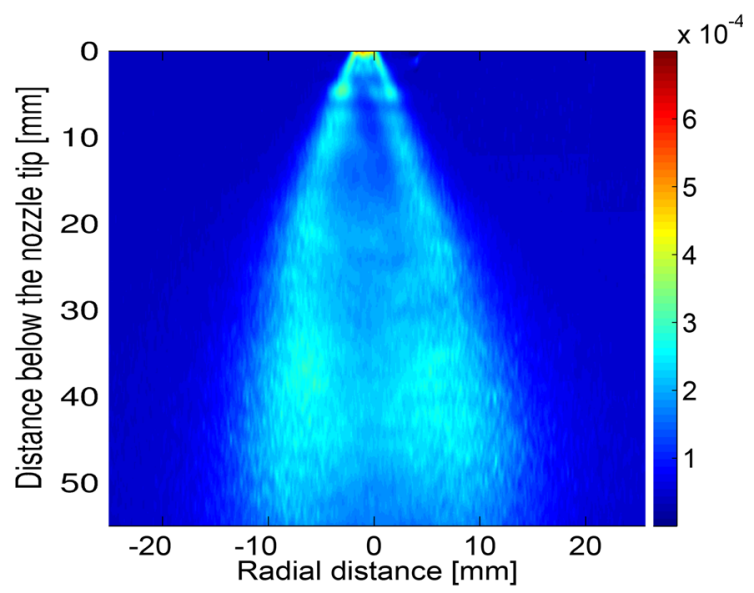

(d) E30 GLR5

Figure 7. Planar liquid volume fraction distributions of SVO and E30 fuels at GLR 1 and 5.

droplets present in the measurement volume, i.e., liquid volume fraction at a point. $K_{1}$ is then used to determine $\rho_{e}$ distribution and liquid volume fraction distribution using Eq. (2).

Figure 6 compares radial liquid volume fraction profiles at $30 \mathrm{~mm}$ below the nozzle tip for the three GLR conditions. The SVO fuel showed a very high liquid volume fraction value $\left(\sim 2.7 \times 10^{-3}\right)$ at GLR 1 due to poor atomization. Most of the liquid is accumulated along the periphery of the spray for all test fuels, which shows the hollow cone spray structure at GLR 1. The difference between liquid volume fractions at the spray axis and spray periphery is reduced with the addition of ethanol. This shows an improvement in liquid volume fraction distribution with the addition of ethanol.

The increase in GLR led to the reduction in liquid volume fractions for all test fuels. However, the difference between the maximum liquid volume fractions of SVO and E30 is reduced at GLR 3. Liquid volume fraction profiles are flat compared with those at GLR 1. This shows the uniform distribution of liquid around the spray axis with the increase in GLR. When GLR is increased further to 5, the liquid is uniformly distributed for E30 blend. The difference in liquid volume fraction of SVO and E30 is small, which suggests marginal improvement in liquid volume fraction with the addition of ethanol at high GLR condition. The liquid volume fraction distribution is an indicator for air-fuel mixture formation, which depicts temperature distribution in a flame. It is observed that at GLR 1 all the test fuels have variation in liquid distribution along the plane. With the addition of ethanol the variation decreases; however, better distribution of the liquid is obtained only at GLR 3 and 5.

\subsection{Planar liquid volume fraction distributions}

Figure 7 compares planar liquid volume fraction distributions of SVO and E30 fuels at GLR 1 and GLR 5 conditions. The liquid volume fraction is high (maximum $\sim 2.7 \times 10^{-3}$ ) for SVO fuel at GLR 1 along the periphery. The spray shows a hollow cone spray structure at this GLR condition. The 
atomizing air has an angular velocity, which might have led to the hollow cone spray structure at GLR 1. The fuel is distributed non-uniformly in the spray plane. Moreover, asymmetry in liquid volume fraction distribution observed at a few locations might be attributed to the presence of random big droplets generated due to the poor atomization of SVO. Addition of ethanol to the SVO fuel has significantly improved the liquid volume fraction distribution at GLR 1 for E30 fuel. At GLR 5 condition, SVO showed more uniform liquid volume fraction distribution compared with that of GLR 1. Most of the liquid is still accumulated around spray periphery, showing semi-solid cone spray structure for SVO fuel at GLR 5. Addition of ethanol results in uniformly distributed liquid, and the lowest liquid volume fraction is observed for E30 fuel at GLR 5 (maximum $\sim 3 \times 10^{-4}$ ). The liquid volume fraction distribution improves with both increase in GLR and addition of ethanol.

The results show that SMD distribution along with the liquid volume fraction should be considered for understanding the spray characteristics. At GLR 1, although atomization improves for E30 blend based on SMD values, the liquid distribution is still non-uniform. More uniform SMD distribution and liquid volume distribution is obtained for GLR 5 and E30 blend. The SMD and liquid volume fraction give a better understanding of air-fuel mixture formation in the combustor.

The spray characteristics are observed to depend on GLR and ethanol blending. It is observed that the addition of ethanol has significant effect at low GLR conditions, whereas marginal improvement in the spray characteristics is observed at high GLR conditions. This might be because of modes of atomization at various GLR conditions. The atomizer might be operating in the classical mode of atomization at GLR 1, where liquid properties have significant control over atomization [38]. In the classical mode of atomization, instability waves are formed along the interface of the liquid mainly due to relative velocity and density gradient between the liquid and the atomizing air. These instability waves are propagated and lead to a disintegration of the liquid. The propagation of the instability waves might be hindered by high viscosity and surface tension of the liquid mainly due to viscous dissipation. Hence, liquid properties have significant control over the atomization in the classical mode. When the GLR is increased, the atomizer might be working on the prompt mode of atomization due to the higher momentum of the atomizing gas [38]. The prompt mode is characterized by instantaneous and violent disruption of the liquid by the atomizing air. The liquid properties have a marginal role in atomization in this mode. The addition of ethanol has strong impact at GLR 1 on drop size distribution and planar SMD distribution. This has an additional advantage that low pumping power of the atomizing air is required while operating the atomizer at low GLR conditions. Therefore, an addition of ethanol at GLR 1 has a benefit of low pumping power of atomizing air with better drop size distribution and more uniform planar SMD distribution.

\section{Summary and conclusions}

An experimental investigation is carried out to study the effect of ethanol blending in Jatropha SVO on planar drop size and liquid volume fraction distributions in airblast sprays. The planar SMD distribution and liquid volume fraction distribution are obtained using a combination of SLIPI-LSD technique and PDIA technique. Numerical corrections are implemented to improve the accuracy of the measurements. The drop size distributions, obtained using the PDIA technique at the periphery of the spray, showed a large number of big droplets for SVO at GLR 1. The drop size distributions for ethanolblended fuels at GLR 1 showed the presence of a large number $(90 \%)$ of small droplets (droplet diameter $30 \mu \mathrm{m})$. The liquid volume fraction of SVO is high at GLR 1 along the spray edges due to poor atomization. The liquid volume fraction distribution improved with ethanol blending at GLR 1. Planar SMD distribution of SVO fuel at GLR 1 shows the presence of big droplets along the spray periphery, which are undesirable in a combustion chamber. E30 fuel showed better SMD distribution compared with that of SVO at GLR 1. A uniform SMD distribution in the range of $40 \mu \mathrm{m}$ is obtained for E30 fuel at GLR 5. The liquid volume fraction of E30 fuel at GLR 5 shows uniformly distributed liquid.

The conclusions of the study are as follows:

- SVO showed poor atomization at GLR 1 due to its high kinematic viscosity.

- Ethanol blending improves drop size distribution, SMD distribution and liquid volume fraction distribution at low GLR (GLR 1) conditions.

- The ethanol blending has a marginal impact on the spray characteristics at high GLR conditions due to prompt atomization.

- E30 fuel showed uniformly distributed liquid in the spray plane with uniform SMD distribution in the range of $40 \mu \mathrm{m}$ at GLR 5.

\section{Acknowledgements}

The authors acknowledge the support from DST-SERB (Grant number: DST SB/S3/MMER/0028) for this work.

\section{References}

[1] Gupta K K, Rehman A and Sarviya RM 2010 Bio-fuels for the gas turbine: a review. Renew. Sust. Energy Rev. 14: 2946-2955

[2] Ma F and Hanna M A 1999 Biodiesel production: a review. Bioresour. Technol. 70: 1-15 
[3] Kang S B, Jin K J and Im Y H 2013 An experimental investigation of a direct burning of crude jatropha oil (CJO) and pitch in a commercial boiler system. Renew. Energy 54: 8-12

[4] Desmira N, Kuniyuki K, Morita S and Gupta A K 2014 Insitu spectroscopic monitoring of jatropha oil combustion properties. Renew. Energy 63: 775-778

[5] Shah P R, Gaitonde U N and Ganesh A 2018 Influence of soy-lecithin as bio-additive with straight vegetable oil on CI engine characteristics. Renew. Energy 115: 685-696

[6] Hashimoto N, Nishida H, Kimoto M, Tainaka K, Ikeda A and Umemoto S 2018 Effects of jatropha oil blending with C-heavy oil on soot emissions and heat absorption balance characteristics for boiler combustion. Renew. Energy 126: 924-932

[7] Lefebvre A 1988 Atomization and sprays. CRC Press, Boca Raton

[8] Fan Y, Hashimoto N, Nishida H and Ozawa Y 2014 Spray characterization of an air-assist pressure-swirl atomizer injecting high-viscosity jatropha oils. Fuel 121: 271-283

[9] Sivakumar D, Vankeswaram S K, Sakthikumar R, Raghunandan B N, Hu J T C and Sinha A K 2016 An experimental study on jatropha-derived alternative aviation fuel sprays from simplex swirl atomizer. Fuel 179: 36-44

[10] Lefebvre A 1980 Airblast atomization. Prog. Energy Combust. Sci. 6: 233261

[11] Basak A, Patra J, Ganguly R and Datta A 2013 Effect of transesterification of vegetable oil on liquid flow number and spray cone angle for pressure and twin fluid atomizers. Fuel 112: 347-354

[12] Avulapati M M and Ravikrishna R V 2015 Experimental studies on air-assisted atomization of jatropha pure plant oil. Atomization Spray. 25: 553-569

[13] Lujaji F C, Boateng A A, Schaffer M, Mtui P L and Mkilaha I S 2016 Spray atomization of bio-oil/ethanol blends with externally mixed nozzles. Exp. Therm. Fluid Sci. 71: 146-153

[14] Simmons B M and Agrawal A K 2012 Flow blurring atomization for low-emission combustion of liquid biofuels. Combust. Sci. Technol. 184: 660-675

[15] Kannaiyan K, Banda M V K and Vaidyanathan A 2016 Planar sauter mean diameter measurements in liquid centered swirl coaxial injector using laser induced fluorescence, Mie scattering and laser diffraction techniques. Acta Astronaut. 123: $257-270$

[16] Betelin V B, Smirnov N N, Nikitin V F, Dushin V R, Kushnirenko A G and Nerchenko V A 2012 Evaporation and ignition of droplets in combustion chambers modeling and simulation. Acta Astronaut. 70: 23-35

[17] Panchasara H V and Agrawal A K 2010 Characteristics of preheated bio-oil sprays produced by an air-blast injector. In: Proceedings of ASME Turbo Expo 2010: Power for Land, Sea, and Air, American Society of Mechanical Engineers, pp. 619-629

[18] Kulkarni A P and Deshmukh D 2019 Improvements in laser sheet dropsizing using numerical and experimental techniques Int. J. Multiph. Flow 110: 273-281, https://doi.org/ 10.1016/j.ijmultiphaseflow.2018.09.004

[19] Jain S, Somasundaram S and Anand T N C 2016 A fluorescent laser-diffuser arrangement for uniform backlighting. Meas. Sci. Technol. 27: 025406

[20] Kulkarni A P and Deshmukh D 2017 Spatial drop-sizing in airblast atomization-an experimental study. At. Spray. 27: 949-961
[21] Anand T N C 2014 PDIA_v0.85 [online]. Available at: https://home.iitm.ac.in/anand/codes/ [accessed on 29-12-2014]

[22] Kashdan J T, Shrimpton J S and Whybrew A 2003 Twophase flow characterization by automated digital image analysis. Part 1: Fundamental principles and calibration of the technique. Part. Part. Syst. Charact. 20: 387-397

[23] Kashdan J T, Shrimpton J S, Booth H J and Whybrew A 2000 Assessment of particle characterisation via phase Doppler anemometry and automated particle image analysis techniques. In: Proceedings of the 10th International Symposium on Application of Laser Techniques to Fluid Mechanics, Lisbon, Portugal

[24] Blaisot J B and Yon J 2005 Droplet size and morphology characterization for dense sprays by image processing: application to the diesel spray. Exp. Fluids 39: 977-994

[25] Kulkarni A P and Deshmukh D 2018 Planar liquid volume fraction measurements in airblast sprays using SLIPI technique with numerical corrections. Appl. Phys. B 124: 187

[26] Perrin W and Tarn W H 1990 CRC handbook of metal etchants. CRC Press, Boca Raton, pp. 106-108

[27] Kristensson E 2012 Structured laser illumination planar imaging: SLIPI applications for spray diagnostics. $\mathrm{PhD}$ Thesis, Lund University, Lund

[28] Kristensson E, Araneo L, Berrocal E, Manin J, Richter M, Aldén M and Linne M 2011 Analysis of multiple scattering suppression using structured laser illumination planar imaging in scattering and fluorescing media. Opt. Express 19: 13647-13663

[29] Berrocal E, Kristensson E, Richter M, Linne M and Aldén M 2008 Application of structured illumination for multiple scattering suppression in planar laser imaging of dense sprays. Opt. Express 16: 17870-17881

[30] Berrocal E, Kristensson E, Hottenbach P, Aldén M and Grünefeld G 2012 Quantitative imaging of a non-combusting diesel spray using structured laser illumination planar imaging. Appl. Phys. B 109: 683-694

[31] Pastor J V, Lopez J J, Juliá J E and Benajes J V 2002 Planar laser-induced fluorescence fuel concentration measurements in isothermal diesel sprays. Opt. Express 10: 309-323

[32] Abu-Gharbieh R, Persson J L, Försth M, Rosén A, Karlström A and Gustavsson T 2000 Compensation method for attenuated planar laser images of optically dense sprays. Appl. Opt. 39: 1260-1267

[33] Deshmukh D and Ravikrishna R V 2013 A method for measurement of planar liquid volume fraction in dense sprays. Exp. Therm. Fluid Sci. 46: 254-258

[34] Holman J P and Gajda W J 2001 Experimental methods for engineers, vol. 2. New York: McGraw-Hill

[35] Vazquez G, Alvarez E and Navaza J M 1995 Surface tension of alcohol water + water from 20 to 50 degree C. J. Chem. Eng. Data 40: 611-614

[36] Deshmukh D 2011 Studies on atomization and sprays of plant oil biofuels using laser-based diagnostics. PhD Thesis, Indian Institute of Science Bangalore, Bangalore

[37] Liu C, Liu F, Mao Y, Mu Y and Xu G 2014 Experimental investigation of performance of an air blast atomizer by planar laser sheet imaging technique. J. Eng. Gas Turb. Power 136: 021601

[38] Lefebvre A 1992 Energy considerations in twin-fluid atomization. J. Eng. Gas Turb. Power 114: 89-96 